\title{
RFID en la gestión y mantenimiento de bibliotecas
}

\author{
Por Alberto Gómez-Gómez, Borja Ena-Rodríguez y Paolo Priore
}

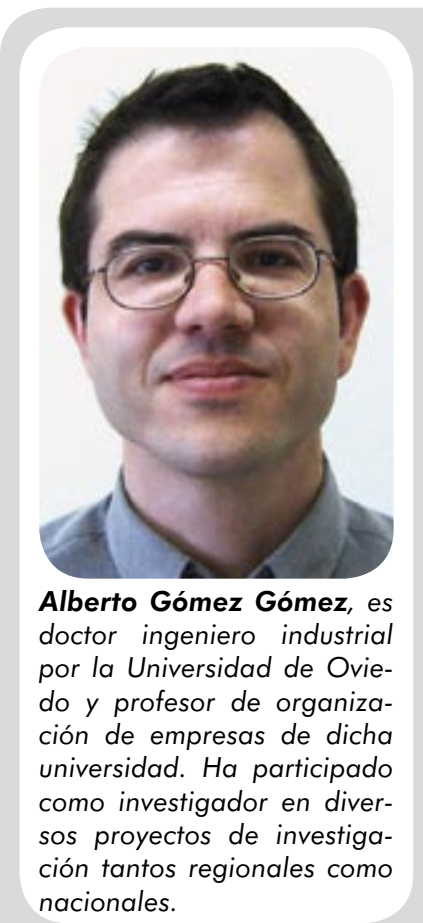

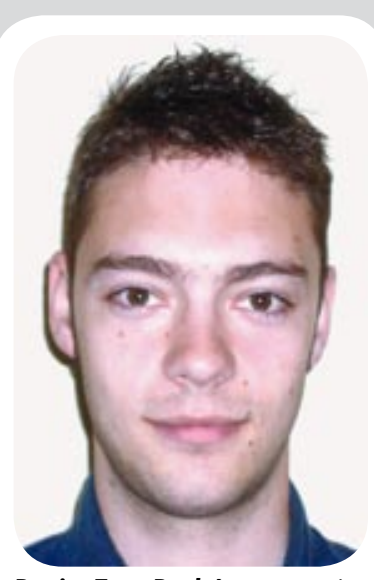

Borja Ena Rodríguez, es ingeniero en telecomunicación por la Universidad de Oviedo. Miembro del grupo de Ingeniería de Organización de la Universidad de Oviedo y becario del proyecto "Análisis y propuesta de mejoras en un sistema de trazabilidad" $^{\prime \prime}$

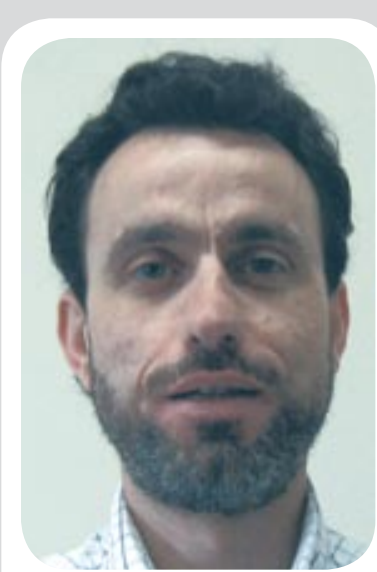

Paolo Priore, es profesor titular de Universidad del Departamento de Administración de Empresas y Contabilidad de la Universidad de Oviedo. Ha participado como investigador en diversos proyectos de investigación tantos regionales, como nacionales e internacionales.

Resumen: La identificación por radiofrecuencia (RFID) es una tecnología emergente que tiene un gran auge en la actualidad. Mediante ella se consigue el almacenamiento, transporte y recuperación de datos en etiquetas que se colocan en productos, para así conseguir una mejora en el control y el seguimiento. En la mayoría de las aplicaciones para bibliotecas se usan etiquetas de RFID a 13,56MHz, regidas por los estándares ISO15693 y ISO18000-3. Este artículo explica de manera simple el funcionamiento de un sistema RFID y sus componentes, citando algunas aplicaciones en diversos sectores, para después centrarse en el sector de las bibliotecas. Se propone un modelo general de implantación de RFID en una biblioteca y se muestran aplicaciones reales ya instaladas, mostrando los beneficios obtenidos por estos centros.

Palabras clave: RFID, Bibliotecas, Gestión, Tecnología, Informática, Identificación por radiofrecuencia.

\section{Title: RFID in library management and maintenance}

Abstract: Radio frequency identification (RFID) is an emergent technology that is currently growing in importance. Companies can improve inventory control and track their products by gathering data from RFID labels on the products. Most of the applications for libraries use RFID of 13.56MHz or less, following ISO standards 15693 and 18000-3. The components and operation of an RFID system are described and applications in diverse sectors are noted, particularly with respect to libraries. A general RFID implementation model for a library is presented and practical applications currently in use are described, along with the benefits that result.

Keywords: RFID, Libraries, Management, Technology, Computer science, Radio frequency identification.

Gómez-Gómez, Alberto; Ena-Rodríguez, Borja; Priore, Paolo. "RFID en la gestión y mantenimiento de bibliotecas". En: El profesional de la información, 2007, julio-agosto, v. 16, n. 4, pp. 319-328.

DOI: 10.3145/epi.2007.jul.05

\section{Introducción}

La identificación, el control y el seguimiento de productos son factores clave en todos los sectores de una sociedad donde los automatismos cobran cada vez más fuerza. Una de las principales tecnologías que impulsan esta automatización es la identificación por radiofrecuencia (RFID). Su propósito es el almacenamiento remoto de datos, el transporte y la identificación de los mismos utilizando etiquetas y lectores. La información guardada en las etiquetas se lee y se procesa según las necesidades concretas de cada aplicación.

En cierto modo, una etiqueta $R F I D$ es similar a un código de barras, aunque presenta sustanciales ventajas como la posibilidad de cambiar la información de la misma, con lo que se puede realizar un seguimiento o historia del producto en cuestión. Además, tiene mayor capacidad de almacenamiento de datos, no precisa de visión directa para leerla y puede operar en entornos 
con polvo, pintura o niebla. Sin embargo, no se debe considerar la RFID como un sustituto del código de barras (probablemente no llegue a sustituirlo nunca, al menos a corto plazo, aunque sí pueda convivir con él) ya que ésa es sólo una de sus aplicaciones.

Los datos almacenados en una etiqueta RFID pueden identificar o localizar el producto, o pueden ser datos específicos como el precio, el color u otras cualidades. Indica en concreto lo que ha sido detectado; por ejemplo, si se incluye una etiqueta en un cd-rom, se asegura que sea localizado ése específicamente, y no otro igual.

Las primeras aplicaciones de este tipo datan de la Segunda Guerra Mundial cuando los británicos usaron el sistema IFF (identification: friend or foe = identificación: amigo o enemigo) para saber si los aviones detectados en el radar eran aliados o no. En las siguientes décadas se usó para localizar robos en grandes almacenes, apertura automática de puertas (Finkenzeller, 2003) o control de peajes. Sin embargo, es en los últimos años donde se ha producido la explosión de la tecnología, debido en gran medida a la creación del estándar EPC (electronic product code = código electrónico de producto). Este código, que normalmente consta de 96 bits, se incluye en una etiqueta RFID. Con él se pueden proveer identificadores únicos para 268 millones de empresas, que podrán tener 16 millones de clases de objetos y 6.800 millones de números de serie en cada clase de objeto (GCI, 2003). Alrededor del $E P C$ se ha creado una arquitectura global para la identificación de cualquier elemento de todo tipo de industria, permitiendo el intercambio de información entre las diferentes empresas.

http://www.epcglobalinc.org

"La consultora Gartner Consulting considera RFID como una de las tecnologías con mayor proyección de crecimiento en los próximos años, según su informe de $\mathbf{2 0 0 5}$ de las nuevas tecnologías emergentes"

La consultora Gartner Consulting considera a $R F I D$ como una de las tecnologías con mayor proyección de crecimiento en los próximos años, según su informe de 2005 de las nuevas tecnologías emergentes (Gartner Consulting, 2005). Su alcance potencial en multitud de aplicaciones no ha pasado inadvertido para nadie. Muchas empresas ven en ella una forma de mejora del rendimiento, de ahorro de costes y de au- mento de la productividad. Mediante RFID se puede conseguir automatización eliminando errores humanos en la identificación, clasificación y seguimiento de objetos; mejoras en la administración de la información y en la calidad de servicio; incremento en la rentabilidad del negocio y en la eficiencia, reduciendo el tiempo de realización de inventarios, y sobre todo disminuyendo los costes de trabajo, operativos y de producción. Ramón Salvía, director general de Sato Iberia opina que llegará a ser la tecnología por excelencia del siglo XXI (Salvía, 2006).

Las bibliotecas siempre han estado al frente de los cambios cuando se trata de información digitalizada a través de redes. Incluso la biblioteca del Vaticano ha etiquetado 120.000 volúmenes con etiquetas RFID (Libbenga, 2004). Las tecnologías inalámbricas están ayudando mucho al desarrollo de una biblioteca inteligente en la que un usuario podría realizar el préstamo y la devolución de libros de manera automática, segura y eficaz, con un control preciso de todas las entradas y salidas de los libros. El actual desarrollo del mercado muestra que la $R F I D$ se está usando con unos 20 millones de libros (Kern, 2004). Entre las principales ventajas de esta nueva tecnología conviene destacar la posibilidad de registrar la entrada/salida de una gran cantidad de libros mediante una única lectura (McCullough, 2003).

En este artículo se hará una breve introducción a esta tecnología, explicando sus principales características y citando algunas de sus actuales aplicaciones en diversos sectores comerciales. Se profundizará después en su uso particular en las bibliotecas, citando un modelo general de aplicación y las ventajas que con ello se obtienen. Por último, se citarán casos concretos de bibliotecas que ya han incorporado sistemas de RFID y se mostrarán las favorables consecuencias que ha supuesto su implantación.

\section{La tecnología RFID}

\subsection{Sistema}

Un sistema RFID se compone de un lector (transmisor/receptor+codificador) que lee y escribe datos en los dispositivos y un transponder (etiqueta de $R F$ ) situado en el objeto que se quiere identificar. Ambos dispositivos tienen una antena incorporada para comunicarse entre sí. El funcionamiento de comunicación es el siguiente:

- El lector genera un campo de radiofrecuencia, normalmente conmutando una bobina a alta frecuencia.

- Este campo genera una corriente eléctrica sobre la bobina de recepción del dispositivo. Esta señal es 
rectificada y de esta manera se alimenta el circuito de la tarjeta.

- Cuando la alimentación es suficiente, la etiqueta puede transmitir sus datos al lector. Normalmente se transmiten como una perturbación en la propia señal emitida por el lector, aunque también puede generar una señal propia.

- Los datos leídos se decodifican y se pasan a un ordenador o dispositivo útil para su procesamiento.

La comunicación entre el lector y la etiqueta se realiza a una frecuencia determinada o con armónicos de esta frecuencia, según el tipo de etiqueta usada en cada aplicación. El alcance, la velocidad y el protocolo empleado para la transmisión dependerán de la etiqueta y la frecuencia elegidas. Existen sistemas que emplean la misma bobina para transmitir la energía a la etiqueta y los datos, así como otros sistemas que utilizan dos bobinas. Estos últimos son más caros pero consiguen mayores prestaciones.

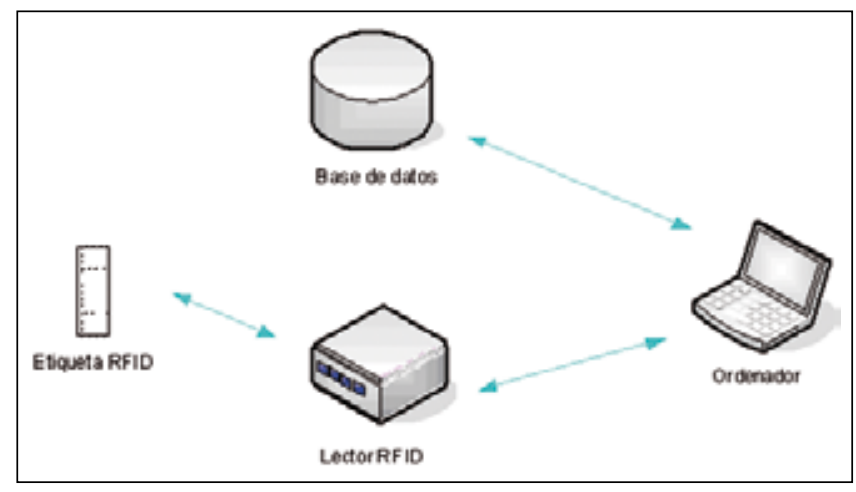

Figura 1. Esquema de un sistema RFID

\subsection{Etiquetas RFID}

Están formadas por un circuito de almacenamiento de datos y una antena para emitir y recibir. Tienen un tamaño variable, desde pocos milímetros hasta varios centímetros y existen de 2 tipos:

Etiquetas pasivas: toman la energía necesaria para transmitir los datos al lector de la propia señal proveniente de éste; así, cuando ha almacenado suficiente energía se produce la transmisión. Sólo funcionan en el rango de alcance del lector. No son capaces de generar señales de alta intensidad y requieren que la que les llegue del lector sea de gran fuerza. Pueden tener un tamaño muy reducido y ser finas como una hoja de papel, con una memoria de unos 128 bytes.

- Etiquetas activas: disponen de una pila o un sistema de alimentación interna, lo que les permite estar siempre en funcionamiento, por lo que no necesitan de una señal de mucha intensidad por parte del lector para poder transmitirle sus datos. Su memoria puede ser de hasta 128 KBytes, gracias a lo cual tienen capacidad de monitorización del entorno, por lo que son frecuentemente usadas junto con sensores en productos de los cuales se quiere saber su historia: temperaturas por las que atraviesa, monitorización del tiempo, etc. Son consecuentemente más caras que las anteriores y el tamaño medio es el de una moneda, aunque hay varias clases, según las aplicaciones.

Tanto unas como otras tienen distintas funcionalidades, y son usadas en las aplicaciones dependiendo del objetivo a conseguir. Las pasivas son más apropiadas en herramientas en las que el movimiento de los productos es constante y controlado, no siendo necesario ni almacenar datos ni monitorizar el entorno. Por su parte, el uso de las activas es una mejor opción en procesos dinámicos, o donde el movimiento de los productos etiquetados es variable y se requiere búsqueda de datos, monitorización del entorno o gran capacidad de almacenamiento de datos. En algún caso puede ser preciso utilizar ambos tipos de tecnología para lograr un nivel de producción y control óptimo.

Se puede realizar otra clasificación según la frecuencia a la que trabajen. Atendiendo a este criterio se tienen los siguientes tipos de etiquetas:

- LF: frecuencia baja (entre 125 y 134,2 KHz). Esta frecuencia está aceptada en todo el mundo y puede operar cerca de metales. Sin embargo, no está aceptada por el EPC, por lo que está cayendo en desuso para aplicaciones de control de productos.

- HF: alta frecuencia $(13,56 \mathrm{MHz})$. La radiación a esta frecuencia no es absorbida por el agua ni por la piel humana, de ahí que se use mucho para la identificación de animales y de barriles con líquidos.

- UHF: frecuencia ultra alta (868 a $956 \mathrm{MHz}$ ). Estas etiquetas no pueden ser utilizadas de forma global porque no existen regulaciones globales para su uso y presentan problemas en determinadas superficies. Actualmente están siendo las más utilizadas, ya que los estándares tienden a estas frecuencias.

- Etiquetas de microondas (2,45 GHz).

Actualmente se tiende a usar sólo etiquetas en frecuencias HF y UHF. La tecnología UHF está menos desarrollada para RFID que la HF, sin embargo, las empresas están optando por la UHF para sus nuevos proyectos, debido en gran medida al estándar EPC. Hay que tener en cuenta que las etiquetas pierden bastante alcance con líquidos y metales. Otra de las limitaciones de $R F I D$ en UHF es la presencia de zonas nulas en el área radiada. También se puede producir la pérdida de alguna etiqueta cuando se encuentran varias relativamente cerca unas de otras. Las ondas de UHF no se ven afectadas en gran medida por ruido de motores o fluorescentes, sin embargo sí se muestran influidas por 
otros sistemas como teléfonos móviles o sondas de radio, aunque la mayoría de estas fuentes emiten en bandas muy estrechas. También existen técnicas para reducir las interferencias, como el uso de FHSS (frequency hopping spread spectrum = espectro ensanchado por salto en frecuencia), distribuyendo la energía a lo largo de la banda de frecuencias, reduciendo así las posibles interferencias creadas a otros sistemas y evitando el bloqueo del receptor. Hoy por hoy se podría decir que $\mathrm{HF}$ es mejor para etiquetar productos a nivel individual, mientras que UHF funciona mejor en etiquetado a nivel de palet*. Aunque siempre hay que tener en cuenta el entorno y la naturaleza del producto a etiquetar a la hora de tomar una decisión entre una frecuencia y otra.

*Un pallet o palet es un armazón de madera o plástico empleado en el movimiento de cargas que facilita su levantamiento y manejo con carretillas elevadoras.

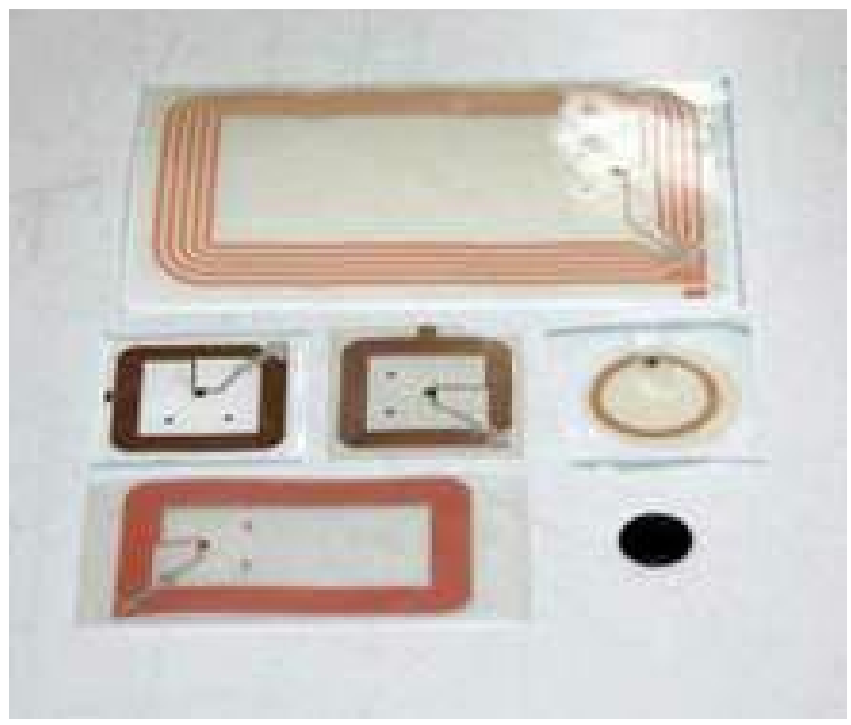

Figura 2. Etiquetas

(Fuente: http://es.wikipedia.org/wiki/RFID)

\subsection{Lectores $R F I D$}

Son los encargados de detectar las etiquetas en su rango de acción y en el caso de que sean pasivas también deben proporcionarles la energía suficiente. Un lector debe ser capaz de acondicionar la señal que recibe, detectar y corregir los errores. Para que sean realmente funcionales deben trabajar a más de una frecuencia (lectores ágiles). Cuando los rangos de acción de varios lectores se solapan se pueden producir colisiones, y para evitar ésto las etiquetas y el lector se comunican mediante un protocolo de forma que sólo la etiqueta adecuada responde al lector.

La mayoría de los lectores incluyen procedimientos para comunicarse con un ordenador ( $R S 232, I 2 C, T T L$, $U S B)$. De esta forma los datos obtenidos con el lector pueden procesarse para hacer de ellos el uso oportuno.
De hecho, ya existe algún teléfono móvil y PDA adaptado para RFID.

\subsection{Aplicaciones de RFID en otros sectores}

La identificación por radiofrecuencia se usa ampliamente en diversos sectores comerciales y empresariales. A continuación se exponen varios ejemplos.

Esta tecnología permite tener un control sobre el suministro farmacéutico, proporcionando una visibilidad total de los medicamentos desde su fabricación hasta el punto de venta. Según un informe de la $O M S$ (2006), la falsificación de fármacos ronda entre un $6 \%$ y un $10 \%$ del mercado mundial. La composición de estos medicamentos puede poner en riesgo la salud, debido a la falta de calidad en su fabricación y a que contienen sustancias distintas a las del original. Mediante RFID el consumidor puede tener total seguridad de que lo que está adquiriendo es auténtico y no entraña riesgos para la salud. Los laboratorios, distribuidores y minoristas también se ven beneficiados al tener un mayor control sobre la trazabilidad de sus productos y sus inventarios.

La fabricación de automóviles requiere un complejo seguimiento y trazabilidad de los componentes y sub-componentes con un 100\% de precisión. Requiere gran flexibilidad y soluciones de fabricación fiables. El mercado cada vez exige plazos de entrega más cortos, de ahí que la trazabilidad y la identificación tengan que ser robustas para poder garantizar un seguimiento de las piezas cuando pasan de un proceso a otro. Las aplicaciones de las etiquetas RFID en el sector de la automoción son muy diversas: en el proceso de fabricación de ruedas, en la carrocería, en los componentes electrónicos y en los sistemas de seguridad del vehículo. Varios fabricantes ya lo han adoptado en sus procesos de fabricación (Srivastava, 2004).

También en los aeropuertos existen aplicaciones claras. Incluyendo estas etiquetas en las maletas, el equipaje puede ser direccionado mediante sensores por toda la cadena, llegando al mismo al avión al que debe ser cargado. También reduce en gran medida las pérdidas y ayuda a la hora de identificar equipaje extraviado.

El transporte público es una de las áreas con mayor potencial. Actualmente las compañías pierden una enorme cantidad de dinero con el sistema de venta de billetes, debido al gasto empleado en fabricarlos y al coste de las máquinas expendedoras. Los sistemas de cobro de billetes electrónicos han de satisfacer requisitos en cuanto a velocidad de lectura, escritura, facilidad de uso y resistencia. RFID cumple con estas premisas. Además, las etiquetas aguantan el frío y la suciedad, por lo que se pueden llevar en una cartera o bolso sin peligro de que se degraden. Las compañías obtienen grandes ventajas: reducción en los costes de máquinas expendedoras de billetes, facilidad de cambiar las 
tarifas, obtención de datos estadísticos, mejor uso del transporte público, supresión de la necesidad de vender billetes, permitiendo ahorro de tiempo y menor distracción para el conductor. No sólo las compañías salen ganando, ya que los usuarios también disfrutarían de nuevas ventajas, por ejemplo no necesitaran llevar dinero en metálico, solamente haría falta tener la tarjeta $R F I D$ con dinero, el sistema reduciría el importe del billete en dicha tarjeta. La automatización del sistema también les supondría un ahorro de tiempo.

Esta tecnología puede aportar grandes beneficios en el almacenamiento, transporte y gestión de documentos. Permitiría optimizar su localización (que a menudo se convierte en una tarea complicada), la realización de inventarios y la mejora del control sobre su entrada/salida. Compañías de diversos tipos se beneficiarían de esto, ya que cualquier empresa almacena gran cantidad de documentación que a menudo no se clasifica y ordena correctamente. Las consecuencias del uso de RFID son manifiestas: eliminación de pérdidas de archivos y reducción del tiempo empleado por el personal en la búsqueda de documentos, lo que repercute en una reducción de costes de operación, disminución de gastos derivados del extravío de documentos, facilidad a la hora de realizar inventarios y mejora del nivel de productividad. Hace posible realizar inventarios de cientos de miles de artículos en días en lugar de meses (Singh, et al., 2006).

\section{RFID y las bibliotecas}

La primera biblioteca que empleó RFID fue the Farmington Community Library en Michigan, en 1999 (Smart, 2004). Desde este primer hito, han sido muchas más las que han realizado pruebas e implantaciones (Singh, et al, 2006). Además de mejorar las operaciones de préstamo y devolución, favorecer el archivo correcto de los libros y aliviar la carga de trabajo de los bibliotecarios, los sistemas RFID prometen proporcionar un mejor control frente al robo, así como de los libros no devueltos. Sin embargo, conviene destacar que en el actual estado de desarrollo, su introducción en las bibliotecas se enfrenta a varios problemas todavía no resueltos. Entre los más significativos destaca la falta de privacidad, la ausencia de estándares y el coste, tanto de los dispositivos como del desarrollo de sistemas RFID. A continuación, se describen los principales componentes necesarios para su implantación y funcionamiento en una biblioteca.

\section{"La introducción en las bibliotecas se enfrenta a varios problemas todavía no resueltos"}

\subsection{Modelo de funcionamiento}

El ejemplo que se expone a continuación se basa en un sistema que las compañías Nedap y $3 M$ proponen para la inclusión de RFID en bibliotecas. No necesariamente se debe seguir siempre este modelo; de hecho, se diseña de acuerdo con la situación y a lo que el cliente requiera, pero sí que es un buen ejemplo del uso que se le podría dar (Fabbi; Vaughan, 2000).

\section{http://www.nedap.es \\ http://www.3m.com}

\subsubsection{Etiquetas}

Todos los productos de la biblioteca (libros, cds, dvds, etc.) se deben etiquetar. Las etiquetas están formadas por un chip y una bobina dispuestos sobre un soporte plástico. Esta combinación se puede integrar en una etiqueta de papel. La etiqueta tiene una memoria de lectura/escritura, uno de cuyos bits (0 ó 1) se destina al control antirrobo. Cuando es leída durante el préstamo, el bit antirrobo cambia de estado y cuando se devuelve el libro se activa de nuevo. Esto supone una gran ventaja en comparación con los sistemas actuales en los que las funciones de registro de datos y seguridad están separadas, con lo que se requiere una operación adicional. En el caso de cds o dvds debido a que no es necesario el contacto directo, las etiquetas se pueden colocar en el interior de la carcasa o incluso integradas en ella.

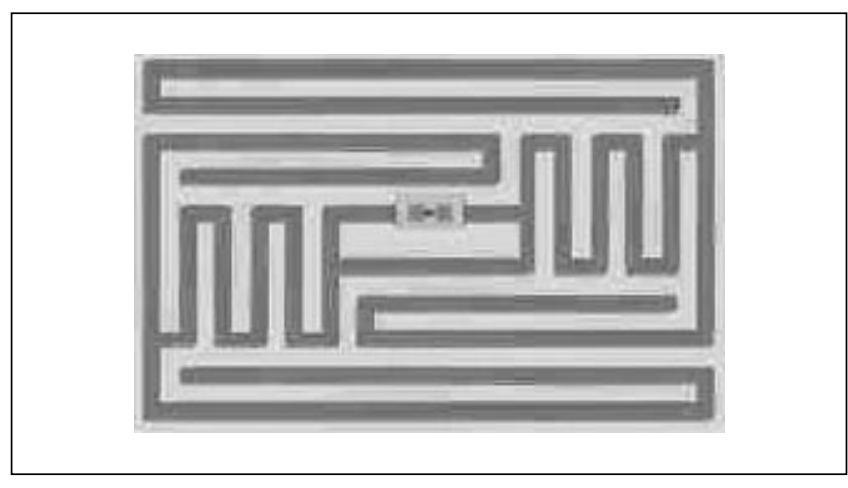

Figura 3. Etiqueta RFID

(Fuente: http://es.wikipedia.org/wiki/RFID)

\subsubsection{Estaciones de préstamo}

El sistema de préstamo está formado por un lector para identificar al usuario, el cual dispone también de una tarjeta que le permite ser identificado fácilmente, un lector RFID sobre el que se sitúan los libros y una pantalla táctil para ayudar en el procedimiento de autopréstamo. Para realizarlo los libros se colocan bajo el lector y la tarjeta del usuario bajo el escáner de código de barras; las etiquetas son leídas automáticamente, desactivando la función antirrobo y quedando registrados en la base de datos. También la tarjeta del usuario 
puede contener una etiqueta $R F I D$, con lo que en ese caso no haría falta lector de código de barras.

\subsubsection{Estaciones de devolución}

La devolución se realiza mediante un lector para ello. Este sistema de auto-devolución se puede usar con el procedimiento de auto-préstamo o implementarlo por separado. Cuando un libro o cd de la biblioteca es devuelto, éste se registra en la base de datos como tal, activándose la función antirrobo a la vez que se leen las etiquetas.

\subsubsection{Lectores portátiles}

Para llevar a cabo inventarios de libros y comprobar la colocación se pueden usar lectores portátiles, consiguiendo así agilizar el trabajo y permitiendo al personal de la biblioteca realizar otras tareas.

\subsubsection{Puertas de seguridad}

El sistema antirrobo está compuesto por dos antenas que forman un pasillo por el cual deben de pasar todos los clientes para entrar y salir de la biblioteca. Cuando un visitante pasa por él con algún artículo sacado incorrectamente con la función antirrobo activada, se dispara una alarma. Numerosos sistemas llevan incorporado un detector de metales para evitar posibles robos en bolsas metálicas.

\subsubsection{Conectividad a la red}

Existe la posibilidad de conectar a internet tanto las antenas como los dispositivos de préstamo y devolución. Esto significa que la información vital de gestión se recoge y procesa directamente. También se puede disponer de un servicio técnico remoto, con el que solucionar los fallos de funcionamiento a distancia.

El software, además de recoger y visualizar información sobre el autoservicio, puede permitir obtener otro tipo de datos, como cantidad y hora de libros y cds prestados, cantidad y hora de libros y cds devueltos, cantidad de alarmas y hora a la que han saltado, cantidad de préstamos no posibles y causas, media de libros prestados por usuario, duración media de los préstamos y comparaciones entre el uso del auto-préstamo y el préstamo atendido.

\subsubsection{Funcionamiento del sistema}

A la entrada y salida de la biblioteca (que puede ser la misma o no) se instalan unas antenas para tener controlados todos los accesos a la misma. El cliente realiza la búsqueda de un libro u otro artículo, para lo que puede pedir la ayuda del personal del centro, realizar una búsqueda en los ordenadores o simplemente mirar en las estanterías.

Una vez que escoge un documento debe ir a la estación de auto-préstamo y colocará su tarjeta de miembro de la biblioteca bajo un lector de código de barras o de $R F I D$, dependiendo del caso. El ordenador le identifica y le muestra sus datos actuales (libros o artículos que tiene en posesión, fecha de devolución, etc.). Si cumple con todas las normas de la biblioteca puede efectuar un préstamo, y para ello pasa el libro por el lector RFID, que le suministra la información a la base de datos y desactiva el bit de seguridad. A la hora de abandonar el centro el cliente debe atravesar los lectores situados a la salida que activarían una alarma en caso de que el libro no haya sido sacado correctamente.

Para devolver los libros simplemente se tienen que dejar en algún contenedor situado al lado de la estación de devolución, previamente leerá la etiqueta de los documentos con el lector para que se actualicen los datos y aparezcan en la base de datos como devueltos, para evitar posibles problemas. También se puede incluir un buzón fuera de la biblioteca para que el usuario deposite el libro a alguna hora en la que está cerrada; si se realiza esto, el personal del centro debe leer siempre la etiqueta de los libros con el lector de la estación de devolución antes de colocarlos en su sitio. Los encargados recogerán los ejemplares devueltos y los colocarán en su lugar.

Para realizar inventarios de libros o para comprobar que todos están situados donde deberían se usan los lectores portátiles. De esta manera un empleado de la biblioteca rastrea las estanterías con el lector, comprobando la correcta situación de los artículos. En el caso de que se busque alguno en concreto, el tiempo de localización se reduce muchísimo frente al que se emplearía en buscar los libros sin ningún sistema de ayuda. El personal de la biblioteca tiene que emplear menos tiempo en este tipo de tareas, con lo que podrá dedicar más a las necesidades de los clientes, que también se ven beneficiados de la mejor ordenación de los libros.

\subsection{RFID y la privacidad}

Una de los temas que a veces surge al hablar de RFID es el de la privacidad. En el caso de una biblioteca, para asegurar este punto se debe mantener la confidencialidad del personal y asegurarse que los procedimientos de lectura son seguros ( $M L A, 2006)$. Hay gente que teme que las etiquetas de sus libros puedan ser leídas y así obtener sus datos. Esto se puede evitar almacenando sólo en las tarjetas de los clientes de las bibliotecas su número de identificación. También existe el temor a que se pueda seguir a una persona que lleve consigo un libro con una etiqueta $R F I D$. Para que no ocurra esto, las bibliotecas pueden tomar medidas de seguridad como desactivarlas al salir o no usar etiquetas de gran alcance.

Desde el punto de vista legal no hay ninguna ley que impida el uso de RFID. Los principales problemas que 
se presentan en este aspecto aparecen cuando se habla de introducir una etiqueta en el documento de identidad, ya que ahí sí que se puede producir una violación de la intimidad de las personas. En los otros casos no existe ningún problema. La protección de la privacidad del consumidor requiere una mezcla de responsabilidades sociales y legales, donde una legislación apropiada es un componente fundamental (Hildner, 2006).

\subsection{Ventajas del uso de RFID}

En la mayoría de las aplicaciones diseñadas para bibliotecas se han empleado etiquetas de HF a 13,56 $\mathrm{MHz}$, ya que no se necesita un gran alcance de lectura y es el estándar más implantado actualmente. Los principales estándares de HF para bibliotecas son el ISO15693 y el ISO18000-3 (ISO, 2001). Las etiquetas son pasivas debido a que la cantidad de información necesaria para almacenar no es muy grande. De hecho, basta con identificar el libro para luego acceder a la base de datos.

\section{"Con RFID los clientes pueden sacar y devolver libros automáticamente"}

Usando RFID se produce una automatización del proceso de préstamo y de devolución, ya que los clientes pueden sacar y devolver libros automáticamente, sin esperas, sacando más de uno a la vez, lo que ahorra mucho tiempo y facilita el sistema. Simplemente acercando los materiales al lector se actualiza la base de datos.

Como se ha dicho, las etiquetas de las bibliotecas suelen venir con un bit antirrobo, que según esté a '0' o a ' 1 ' activará una alarma o no a la salida de la biblioteca, asegurando así que sólo los usuarios autorizados retiren libros. Las pérdidas se reducen enormemente. También se gana tiempo al hacer simultáneamente la lectura del código y la activación/desactivación del bit antirrobo.

A la hora de realizar el inventario de los libros no hace falta ir sacándolos uno por uno, sino que pasando un lector RFID portátil por las estanterías se van detectando los libros que hay en la biblioteca. Además se comprueba su correcta situación por si alguno de ellos estuviera desubicado.

Para conseguir estadísticas fiables sobre el uso de libros o bibliotecas se pueden instalar sensores en los puntos de lectura, para saber qué es lo que más interesa a los usuarios o qué tipos de lectura se debe fomentar.

Sin embargo, aunque ofrece grandes beneficios, el coste de la implantación y los numerosos obstáculos tecnológicos debidos a las dificultades propias de la adaptación de una nueva tecnología pueden ser un problema. La apuesta de los principales desarrolladores de software y hardware, como Microsoft, SAP o Intel, junto con el interés de grandes superficies como Wal Mart o la alemana Metro es un primer gran paso. A medida que las compañías vayan adoptando estas soluciones el mercado crecerá de forma exponencial.

La medición de su coste en comparación con el rendimiento tiene la misma forma que la curva de oferta y demanda típica de economía. A medida que la curva de rendimiento sube, existe un punto en el que el beneficio suministrado por el uso de las etiquetas RFID comienza a superar su coste. A partir de ese momento la adopción de la identificación por radiofrecuencia comienza a tener sentido comercial.

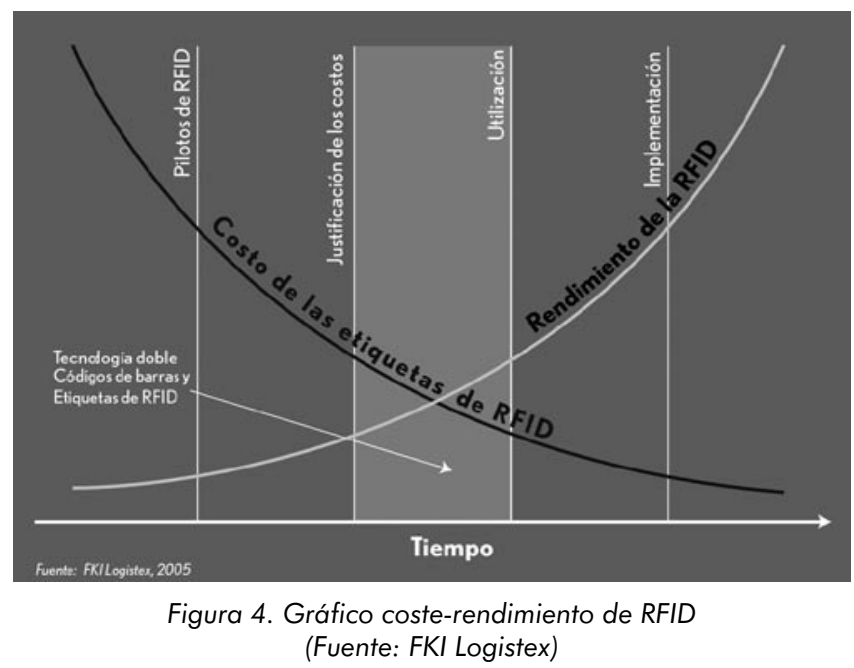

Se deben estudiar los beneficios totales que la implantación acarrearía en una compañía. No se debe valorar sólo el coste de la etiqueta, sino el gasto relativo que ésta tiene y los beneficios (económicos y de gestión) que proporcionaría. Por ejemplo, en muchas aplicaciones de fabricación una etiqueta de $10 €$ puede resultar muy barata si se la compara con el coste del problema que puede solucionar. En cualquier caso, la situación específica debería determinar el coste adecuado de la etiqueta que permitiera tener éxito. A medida que la identificación por radiofrecuencia se adopte de manera más generalizada, el coste de las etiquetas caerá como sucede con todos los nuevos productos cuyo uso se masifica. Es sólo cuestión de tiempo que se vuelva completamente accesible y todo indica que su uso crecerá con el paso del tiempo. Es posible que exista una gran inversión en software, servicios y lectores. Según se vayan aplicando las normas de forma uniforme el gasto se destinará a las etiquetas, y conforme se vayan implantando el coste se irá reduciendo. 


\section{"A medida que la identificación por} radiofrecuencia se adopte de manera generalizada, el coste de las etiquetas caerá rápidamente"

Aunque para realizar el cambio de etiquetaje con código de barras a etiquetas RFID se precisa un tiempo de convivencia de ambas tecnologías (Wu, 2006), en el caso de una biblioteca el procedimiento es relativamente sencillo.

\subsection{Aplicaciones reales de RFID en bibliotecas}

Hasta los últimos años, las implantaciones de estos sistemas eran en su mayoría pruebas piloto (Fabbi, et al., 2002). Sin embargo, en la actualidad, ya se está pasando a un funcionamiento real. En general, los pasos a seguir suelen ser:

- Etiquetado de libros.

- Instalación de lectores, puntos de préstamo y devolución.

- Generación de software adecuado para la gestión, control y mantenimiento de los datos de los clientes y de la localización de los libros, permitiendo la adquisición de datos en tiempo real. lución.

- Implementación de auto-préstamo y auto-devo-

La siguiente tabla da una idea del tiempo necesario para etiquetar todos los libros según las estaciones de las que se disponga:

A continuación se comentan algunos ejemplos reales de funcionamiento, para ilustrar las ventajas de estos nuevos sistemas. Se muestra con mayor detalle el caso de la Biblioteca Nacional de Corea.

- Biblioteca pública West Bloomfield Township: en el transcurso de un año se etiquetaron alrededor de 220.000 libros y cds. Se instalaron 6 estaciones de préstamo en la biblioteca principal y 3 en la secundaria. Tras varios meses de operación, el sistema de autopréstamo llegó a porcentajes superiores al 90\%. Los clientes están satisfechos y el número de alarmas de seguridad se ha reducido.

http://checkpointsystems.com/docs/cpt-west_ bloomfield_library.pdf

- Cerritos Library (California): se decidió optar por RFID para incrementar la productividad del personal, así como proporcionar ventajas a los clientes. Se instalaron etiquetas en todos los libros, cinco estaciones de auto-préstamo y cuatro estaciones para el personal en el escritorio principal para operaciones de auto-préstamo y auto-devolución. Una docena de lectores fueron instalados en varias entradas de la biblioteca. Otros lectores adicionales se pusieron en áreas traseras para facilitar la ordenación de los libros y la realización de inventarios, además se dotó de un lector portátil para una rápida y eficiente búsqueda de libros. Tras un año, el volumen de visitantes por día se había incrementado un $30 \%$ y la circulación anual más del doble. Además, el personal tenía más tiempo para realizar otro tipo de tareas de mantenimiento y cuidado de la biblioteca.

\section{http://checkpointsystems.com/docs/CPT-Cerritos.} $p d f$

- Biblioteca Colchester: se implantó un sistema RFID basado en el estándar ISO 15693. Las etiquetas cuentan con un bit de seguridad para evitar robos. El sistema de autoservicio permite a los clientes sacar libros y devolverlos de manera fácil, cómoda, rápida sin necesitar la presencia de personal. Se diseñó un software específico para tal efecto, lo más amigable posible para los usuarios y se implantó un buzón a la salida de la biblioteca, para que los clientes pudieran devolver los libros a cualquier hora sin tener que entrar dentro de la biblioteca. En el momento que se mete un libro en el buzón, se lee su etiqueta $R F I D$ y el sistema se actualiza dinámicamente, para tener control absoluto de los préstamos y devoluciones. Todo ello está activo desde agosto de 2003, siendo en ese momento la mayor instalación de este tipo en una biblioteca pública del Reino Unido.

http://www.intellident.co.uk/ downloads/ColchesterCaseStudy.pdf

- Biblioteca del Centro de Humanidades y Ciencias Sociales de Madrid: el Ministerio de Educación y Ciencia anunció el 29 de mayo de 2006 la apertura del concurso para implantar un sistema RFID que permita el autopréstamo y la devolución, el control de inventar-

\begin{tabular}{|c|c|c|c|c|}
\hline \multirow{2}{*}{$\begin{array}{c}\text { Tiempo necesario para completar las labores } \\
\text { de etiquetado (en semanas) }\end{array}$} \\
\hline $\begin{array}{c}\text { No de } \\
\text { estacio- } \\
\text { nes }\end{array}$ & \multicolumn{5}{|c|}{ No de libros } \\
\cline { 2 - 5 } & $\mathbf{5 0 . 0 0 0}$ & $\mathbf{2 5 0 . 0 0 0}$ & $\mathbf{5 0 0 . 0 0 0}$ & $\mathbf{7 5 0 . 0 0 0}$ \\
\hline 1 & 10 & 50 & 100 & 150 \\
\hline 2 & 5 & 25 & 50 & 75 \\
\hline 3 & 3,5 & 17 & 34 & 51 \\
\hline 4 & 2,5 & 12,5 & 25 & 37,5 \\
\hline 5 & 2 & 10 & 20 & 30 \\
\hline 6 & 1 & 5 & 10 & 15 \\
\hline
\end{tabular}

Tabla 1. Tiempo de etiquetado (Fuente: Tagsys) 
ios, el suministro de carnets y etiquetas, que tenga un sistema antirrobo en la biblioteca del Centro de $\mathrm{Hu}$ manidades y Ciencias Sociales de Madrid.

http://www.rfid-magazine.com/noticias/detalle. php?id=454

Se ha establecido un plazo de entrega de 6 meses y medio, siendo el importe máximo de $740.000 €$. El sistema requerido se compone de equipos de autopréstamo y devolución, terminales móviles RFID, puertas antirrobo, impresoras y etiquetas de varios tipos, así como de un sistema de gestión de inventario. La frecuencia elegida para el proyecto es $13,56 \mathrm{MHz}$. Se calcula que se necesitan 400.000 etiquetas para libros, 80.000 para revistas y 5.000 para cds y dvds, además de 1.000 carnés para los usuarios de la biblioteca.

\subsubsection{Biblioteca Nacional de Corea, Seul}

La infraestructura de información y telecomunicaciones en Corea del Sur siempre ha sido una de las principales del mundo. El gobierno coreano decidió implantar sistemas $R F I D$ en varios centros, incluida su biblioteca nacional $(N L K)$, la cual tenía varios objetivos, así como diferentes normas internas.

- La NLK no permite a sus clientes sacar material de la biblioteca fuera de sus instalaciones, ya que es una institución que preserva las fuentes intelectuales de la nación. Los materiales almacenados en armarios cerrados se etiquetan con RFID.

- La $N L K$ necesita establecer un sistema para la gestión de la información para ayudar al crecimiento de la biblioteca. Se precisa un sistema que posibilite la obtención de información en tiempo real. Introduciendo un sistema RFID conectado con el sistema de clientes de la biblioteca y con el de información se consigue mejorar la gestión de la información.

- La biblioteca proporciona servicios de información a sus clientes de acuerdo con sus preferencias; por lo tanto es necesario saber cuáles son éstas.

Tras realizar el etiquetado de los artículos se puso en marcha un sistema de gestión de la información

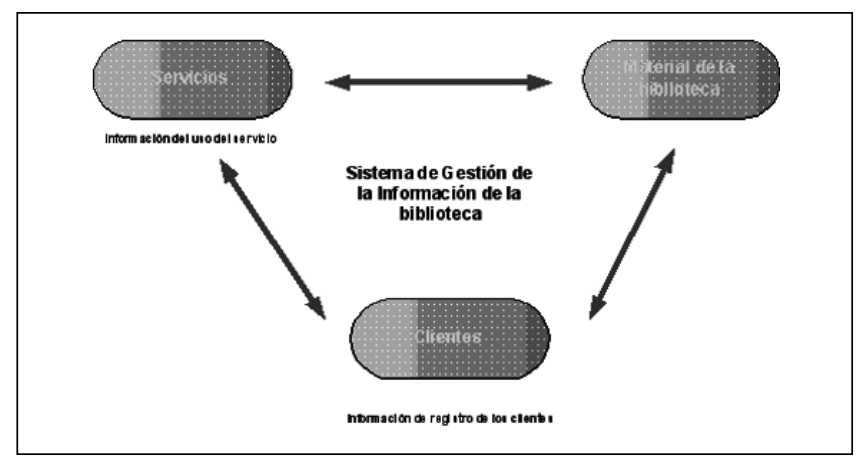

Figura 5. Gestión de la información (Tomado de Sue, 2006) comunicado con el sistema de clientes y con el de información de librerías coreanas. El etiquetado de los 30.000 artículos se efectuó en 2003, y se diseñó un plan desde septiembre de 2004 hasta agosto de 2006 para tenerlo completamente operativo. En 2005 ya se empezaron a recoger estadísticas sobre el flujo de libros en cada sección de la biblioteca.

Se idearon dos tipos de servicios: orientados a los clientes que iban físicamente al centro, y a aquellos que accedían remotamente a través de internet. Así, los primeros podían usar su tarjeta y colocar el libro seleccionado (etiquetado con la etiqueta $R F I D$ ) junto al lector, de esta manera tan práctica y cómoda se realizaba el préstamo. Para aquellos artículos que no se marcaron con etiquetas RFID se diseñó un sistema de búsqueda que facilitaba su retirada. En cuanto a los clientes que accedían a través de internet, se desarrolló la opción 'Mi biblioteca', que evitaba que tuvieran que introducir información cada vez que accedían. Además, también permitía tener un control sobre las consultas que se realizaban en la base de datos para mejorar el funcionamiento de la biblioteca. 'Mi biblioteca' proporcionaba información de los materiales disponibles y buscaba información en la página. A través de este sistema se podían obtener datos sobre libros más consultados, áreas más sugeridas, etc.

El sistema de gestión permite tener datos sobre el flujo de libros, el uso de los sistemas de información de los usuarios y datos estadísticos sobre el número de clientes de la biblioteca en tiempo real, lo que pueden usar los encargados de la biblioteca para mejorar su funcionamiento. Gracias al etiquetado con RFID, la realización de inventarios o la búsqueda de algún libro se han vuelto más sencillas.

Para poder unir el sistema RFID con las diferentes bases de datos se diseñó un formulario de registro de los clientes y una tarjeta de funcionamiento ligada a RFID. Así, cuando un visitante accede a la biblioteca rellena un formulario para conseguir una tarjeta identificativa, que incluye el nombre del cliente y un número. En el patrón de identificación se realizan preguntas acerca de sus gustos para realizar estadísticas. En otra máquina se pueden obtener las tarjetas de manera automática introduciendo su número de identificación.

Las etiquetas usadas fueron pasivas a una frecuencia de $13,56 \mathrm{MHz}$ y su coste fue una de las principales inquietudes cuando se decidió introducir el sistema. Sin embargo, se considera que el elevado grado de desarrollo tecnológico de Corea, junto con el desarrollo de la tecnología RFID permitirá que el coste sea menor en el futuro.

Dejando a un lado este dato, el otro reto era lograr un cambio rápido de tecnología, es decir, introducir 


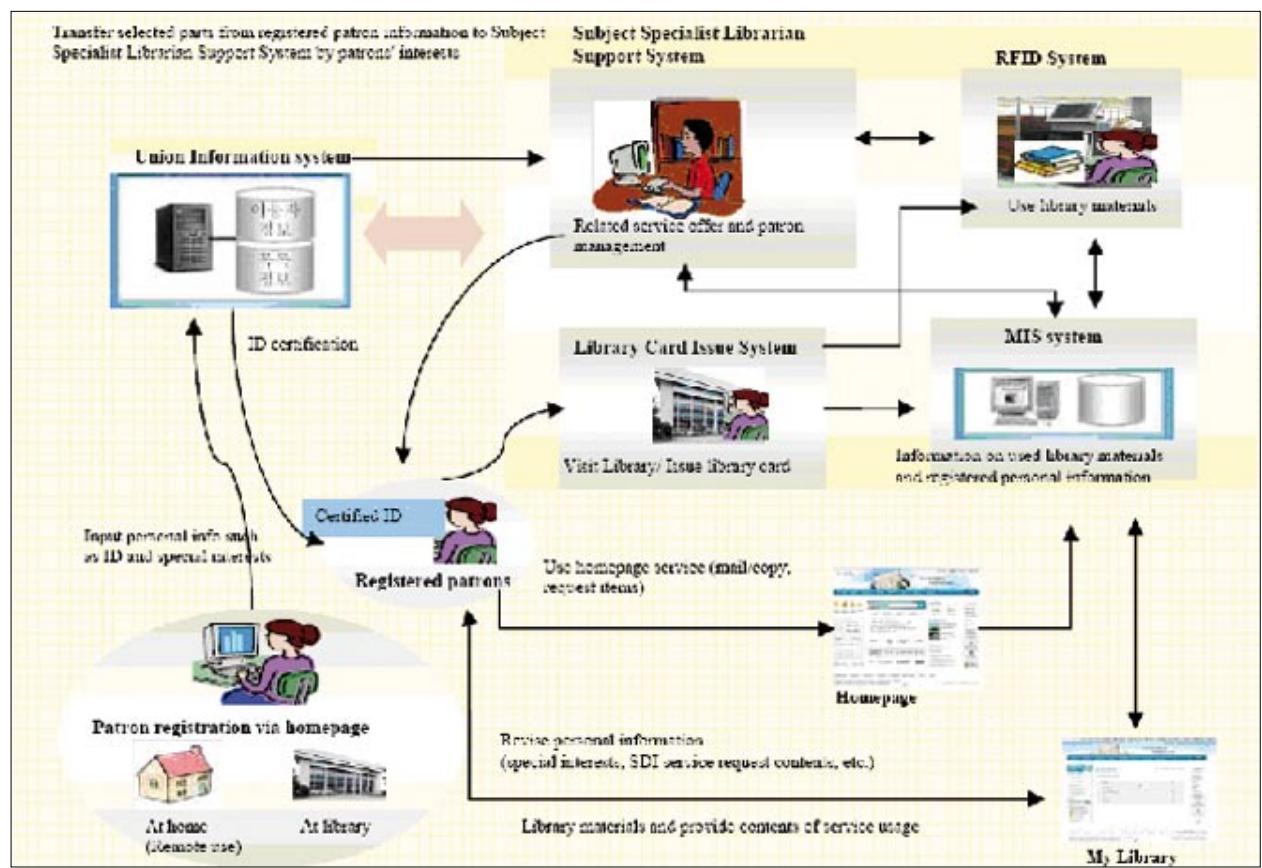

Figura 6. Funcionamiento de la biblioteca (Fuente: http://www.ifla.org)
Gartner Consulting. Gartner highlights key emerging technologies in 2005 hype cycle. Consultado en: 1411-06.

http://www.gartner.com/press_releases/asset_1344 60_11.html

$G C I$ \& IBM. "Mapa de ruta de la Global Commerce Initiative". En: Working paper, 2003. Consultado en: 14-11-06.

http://www.iaccolombia.org/site epc/Banco_de_Informacion/GCI_ Roadmap.pdf

Hildner, N. "Defusing the threat of RFID: protecting consumer privacy through technology-specific legislation at the state level". En: Harvard civil rights-civil liberties law review, 2006 , v. 41 , n. 1, pp. 133-173

International Standards Organization. ISO-Standard 15693: Part 1: Physical characteristics - Part 2: Air interface and initialization - Part 3: Anti-collision and transmission. Consultado en: 30-10-06. http://www.iso.org

Kern, C. "Radio-frequency-identification for security and media circulation in libraries". En: The electronic

rápidamente el nuevo modelo sin perjuicio para los clientes ni para la biblioteca. Los usuarios que no solicitaban préstamos de libros, sino que solamente los necesitaban para el uso interno de la biblioteca podían acercar por error o sin querer su tarjeta o un libro al lector, apareciendo el libro como prestado. Para que no ocurriera ésto, se instalaron estaciones de auto-chequeo, para chequear libros que sólo iban a ser usados dentro de la biblioteca (Sue, 2006).

\section{Conclusiones}

Este artículo se ha realizado analizando las principales características de los sistemas RFID, indicando las particularidades que los hacen especialmente atractivos para la mejora del funcionamiento de las bibliotecas. Mediante esta tecnología se consiguen mejoras significativas en las diferentes actividades relacionadas con la gestión de documentos. Se propone un modelo general de implantación en una biblioteca y se muestran aplicaciones reales ya instaladas, citando los beneficios obtenidos por estos centros.

\section{Bibliografía}

Fabbi, L. J.; Vaughan, J. "The 3M digital library assistant: observations from the field". En: Library computing, 2000, v. 19, n. 1-2, pp. 8-52.

Fabbi, L. J.; Watson, S. D.; Marks, K. E. "Implementation of the 3M Digital Identification System at the UNLV libraries". En: Library hi tech, 2002, v. 20, pp. 104-110.

Finkenzeller, K. "RFID-handbook: fundamentals and applications in contactless smart cards and identification". New York, NY: John Wileys \& Sons, $2^{\text {nd }}$ ed., 2003. ISBN 3446220712. library, 2004, v. 22, n. 4, pp. 317-324.

Libbenga, J. Vatican library adopts RFID, 2004. Consultado en: 30-1006.

Mccullough, J. "Redesigning library applications for PDAs: ILS vendor perspective". En: Library hi tech, 2003, n. 21, pp. 393-399.

MLA. "MLA intellectual freedom manual". Consultado en: 28-10-06. http://www.mdlib.org/divisions/ifap/privacy.pdf

OMS. "Medicamentos falsificados". Nota descriptiva OMS 275, 2006. Consultado en: 14-11-06.

http://www.who.int/mediacentre/factsheets/fs275/es/index.html

Salvía, R. "La tecnología RFID en la industria textil: HF o UHF". En: RFID-magazine, 2006. Consultado en: 14-11-06.

http://www.rfid-magazine.com/opinion/index.php?id0586

Singh, J.; Brar, N.; Fong, C. "The state of RFID applications in libraries". En: Information technology and libraries, 2006, n. 25, pp. 24-32.

Smart, L. The market place; Laura Smart gives a guide to the leading providers of integrated RFID solutions. 2004. Consultado en: 30-10-06. http://mythic.lib.calpoly.edu:2067/universe/document?_ $m=a a 31 c 69 e a 270 d 87 b 08$

Srivastava, B. "Radio frequency ID technology: the next revolution in SMC". En: Business horizons, 2004, v. 47, n. 6, pp. 60-68.

Sue, K. "The implementation of the RFID system for improving customized service: the case of the National Library of Korea". En: World library and information congress, 2006. Consultado en: 27-10-06. http://www.ifla.org/IV/ifla72/papers/140-Kim-en.pdf

Wu, N. C.; Nystrom, M. A.; Lin, T. R.; Yu, H. C. "Challenges to global RFID adoption”. En: Technovation, 2006, v. 26, n. 12, pp. 1.371-1.323.

\section{Alberto Gómez-Gómez, Borja Ena-Rodríguez, Paolo Priore, Universidad de Oviedo.}

agomezg@uniovi.es

borjaena@telecable.es

priore@epsig.uniovi.es http://ww.theregister.co.uk/2004/07/09/vatican_library_rfid 